Research paper

\title{
Investigating nucleo-cytoplasmic shuttling of the human DEAD-box helicase DDX3
}

\author{
Ruth Brennan ${ }^{\mathrm{a}}$, Antje Haap-Hoff ${ }^{\mathrm{b}}$, Lili Gu ${ }^{\mathrm{a}}$, Virginie Gautier ${ }^{\mathrm{c}}$, Aideen Long ${ }^{\mathrm{b}}$, Martina Schröder ${ }^{\mathrm{a}, *}$ \\ a Institute of Immunology, Biology Department, Maynooth University, Maynooth, Co. Kildare, Ireland \\ b School of Medicine, Trinity College Dublin, Trinity Biomedical Sciences Institute, 152-160 Pearse Street, Dublin 2, Ireland \\ ${ }^{\mathrm{c}}$ School of Medicine, Centre for Research in Infectious Diseases (CRID), University College Dublin, Belfield, Dublin 4, Ireland
}

\section{A R T I C L E I N F O}

\section{Keywords:}

Nucleo-cytoplasmic shuttling

Nuclear import

Nuclear export

Cell cycle

DEAD-box helicase

DDX3X

\begin{abstract}
A B S T R A C T
The human DEAD-box helicase DDX3 is a multi-functional protein involved in the regulation of gene expression and additional non-conventional roles as signalling adaptor molecule that are independent of its enzymatic RNA remodeling activity. It is a nucleo-cytoplasmic shuttling protein and it has previously been suggested that dysregulation of its subcellular localization could contribute to tumourigenesis. Indeed, both tumour suppressor and oncogenic functions have been attributed to DDX3. In this study, we investigated the regulation of DDX3's nucleocytoplasmic shuttling. We confirmed that an N-terminal conserved Nuclear Export Signal (NES) is required for export of human DDX3 from the nucleus, and identified three regions within DDX3 that can independently facilitate its nuclear import. We also aimed to identify conditions that alter DDX3's subcellular localisation. Viral infection, cytokine treatment and DNA damage only induced minor changes in DDX3's subcellular distribution as determined by High Content Analysis. However, DDX3's nuclear localization increased in early mitotic cells (during prophase) concomitant with an increase in DDX3 expression levels. Our results are likely to have implications for the proposed use of (nuclear) DDX3 as a prognostic biomarker in cancer.
\end{abstract}

\section{Introduction}

DDX3 is a member of the DEAD-box family of RNA helicases which has been implicated in a wide variety of processes that require RNA remodeling, including splicing, mRNA export, transcriptional and translational regulation, RNA decay and ribosome biogenesis (Schröder, 2010). Eukaryotic cells spatially segregate transcription (occurring in the nucleus) and mRNA translation (occurring in the cytoplasm), allowing for post-transcriptional regulation of gene expression. DDX3 has been implicated in both nuclear and cytoplasmic steps of gene expression (e.g. regulation of transcription and translation), albeit for distinct sets of genes. For example, DDX3 has been suggested to regulate transcription of IFN $\beta$, p $21^{\text {waf1/cip } 1}$ and E-Cadherin, with the ability to either increase or decrease activation of their promoters (Botlagunta et al., 2008; Chao et al., 2006; Soulat et al., 2008). On the other hand, there is accumulating evidence for an important role for DDX3 in the regulation of translation for specific mRNA transcripts (Lai et al., 2010, 2008, 2015; Soto-Rifo et al., 2012, 2013). In addition, we and others have identified non-conventional functions of DDX3 as a signalling adaptor, e.g. in the signalling pathways downstream of antiviral pattern recognition receptors and in the death receptor signalling pathway (Cruciat et al., 2013; Gu et al., 2013, 2017; Oshiumi et al., 2010; Sun et al., 2008). Since DDX3 therefore has multiple distinct nuclear functions (transcription, mRNA processing) and cytoplasmic functions (translation and signalling), it is conceivable that a change in its subcellular localisation could affect its participation in these processes with consequences for cellular function. We were therefore interested in investigating the regulation of its nuclear import and export. It has already been demonstrated that DDX3 shuttles between the nucleus and the cytoplasm; and both CRM-1 (the main protein exporter) and TAP (the main mRNA exporter) have been implicated in its export from the nucleus (Frohlich et al., 2016; Heerma van Voss et al., 2017b; Lai et al., 2008; Schroder et al., 2008; Yedavalli et al., 2004). CRM-1 mediates export of proteins containing a conserved nuclear export

\footnotetext{
Abbreviations: A, area; aa, amino acid; BF, brightfield; CRM-1, chromosome maintenance region-1; DAPI, 4'6-diamidino-2-phenylindole; DDX3, DEAD-box helicase

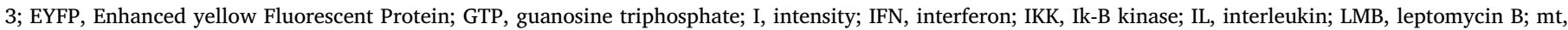

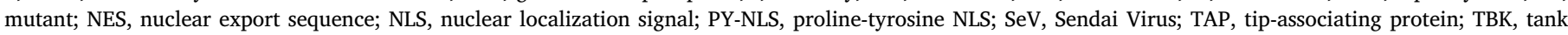
binding kinase; TNF, tumour necrosis factor; wt, wildtype

* Corresponding author.

E-mail address: martina.schroeder@mu.ie (M. Schröder).
} 
sequence (NES) comprised of evenly spaced hydrophobic residues; with leucine usually being most abundant (la Cour et al., 2004). Binding of NES-containing proteins to CRM-1 is controlled by RanGTP, which predominantly localises to the nucleus. While DDX3 contains a conserved leucine-rich NES at its N-terminus, it is controversial whether this NES is required for nuclear export of all DDX3 orthologues. In the Xenopus DDX3 orthologue An3, the NES was clearly demonstrated to be required for its nuclear export, as mutation of two crucial residues in the NES, leucines 19 and 21, prevented export of An3 (Askjaer et al., 1999, 2000). However, the same mutations to the NES did not prevent export of hamster DDX3 (Sekiguchi et al., 2004). For human DDX3, Yedavalli et al. showed binding to CRM-1 in an NES and Ran-GTP-independent manner. They suggested that human DDX3 instead interacts with CRM1 through a region in its C-terminus and that it acts as a CRM1 co-factor in the rev-dependent export of HIV-1 RNAs, rather than regular CRM-1 cargo (Yedavalli et al., 2004). In this paper, we clarify that the N-terminal conserved NES in human DDX3 is functional and required for its export from the nucleus.

In contrast to its export, the determinants of DDX3's nuclear import have not been investigated before. In this study, we therefore screened for nuclear localization signals (NLSs) in the DDX3 sequence and demonstrate that DDX3 contains at least three distinct NLS sequences that can independently mediate nuclear import of DDX3.

Finally, we also aimed to identify factors that alter the nuclear-cytoplasmic distribution of DDX3. Most studies have described a predominantly cytoplasmic localization of DDX3 in the steady state, with it accumulating in the nucleus only when export is inhibited, indicating that it constantly shuttles between the cytoplasm and the nucleus. It has also been suggested that its predominant localization is altered during malignant transformation of cells, and that mis-localisation of DDX3 could even contribute to malignancy. It was suggested that DDX3 is mainly cytoplasmic in squamous cell carcinoma (SCC) cells, but nuclear in normal epidermal cells (Chao et al., 2006). In contrast to this, a more recent study demonstrated that high nuclear DDX3 expression (defined as it being present in more than $1 \%$ of cells) correlated with worse patient outcome, larger tumour size, positive lymph nodes and higher grade cancers in cohorts of breast cancer and colon cancer patients (Heerma van Voss et al., 2017b).

In light of this potential link between DDX3's subcellular localization (or its mis-localisation) and cancer cell malignancy, it is highly relevant to identify the factors that influence the subcellular distribution of DDX3. Here, we demonstrate that DDX3's expression and localization is regulated during cell cycle progression.

\section{Materials and methods}

\subsection{Plasmids and antibodies}

The mammalian expression constructs pCMV-HA-DDX3 and pCMVMyc-DDX3 have been described in our previous studies (Schroder et al., 2008; Gu et al., 2013). pCMV-HA-DDX3 point mutations were introduced following manufacturer's instructions for the QuikChange Site-Directed Mutagenesis Kit (Agilent), except for using Pfusion ${ }^{\mathrm{TM}} \mathrm{HiFi}$ polymerase (New England Biolabs). DDX3 truncation mutants (1-408, 409-662, 130-662, 139-662, 139-408, 22-662, 44-662) were generated by amplifying the relevant sequences by PCR from Ha-DDX3 and inserting them into the pCMV-HA vector. NES-addition mutants, which re-attached the NES region to N-terminal deletion mutants, were generated by amplifying the first 1-22 amino acids of DDX3 flanked by two EcoR1 site. Constructs for truncation mutants were linearised using EcoR1 and the NES 1-22 fragment was ligated into the EcoR1 site. EYFP-tagged DDX3 constructs were generated by sub-cloning the relevant DDX3 sequences into a pCDNA3.1 vector containing the open reading frame for EYFP. All generated constructs were sequence verified.

The antibodies used were anti-Myc mAb clone 9E10 (Sigma-
Aldrich), anti-HA mAb (Covance), anti-DDX3 (A300-474A, Bethyl Laboratories), anti-CRM-1 (Novus Biologicals), anti-Ha AlexaFluor 594 (Invitrogen), anti-mouse AlexaFluor 488 (Invitrogen), anti-mouse AlexaFluor 594 (Invitrogen), anti-rabbit AlexaFluor 488 (Invitrogen) and anti-rabbit AlexaFluor 594 (Invitrogen), Cyclin A, B, and E (all Santa Cruz).

\subsection{Cell culture and transfection}

HeLa and HEK293T cells were maintained in Dulbecco's modified Eagle's medium (DMEM) with Glutamax ${ }^{\mathrm{TM}}$ (Gibco) supplemented with $10 \%$ fetal calf serum and $50 \mathrm{ng} / \mathrm{ml}$ gentamycin (Sigma-Aldrich). Transient DNA transfections for immunofluorescent staining and immunoprecipitations/pull-downs were performed using the Calcium Phosphate method. Transient transfection for high content analysis was performed using GeneJuice ${ }^{\mathrm{mt}}$ (Merck/Novagen). Total amount of DNA was kept constant by addition of empty vector.

\subsection{CRM-1 Co-immunoprecipitation}

Co-immunoprecipitations were performed on cell lysates of transiently transfected HEK293T cells, which were harvested $24 \mathrm{~h}$ after transfection. Cells were lysed in IP lysis buffer (50 mM Hepes pH7.4, $150 \mathrm{mM} \mathrm{NaCl}, 2 \mathrm{mM}$ EDTA, 1\% NP-40, 10\% Glycerol, $10 \mathrm{mM} \mathrm{NaF}, 1 \%$ Triton X-100, $10 \mathrm{mM}$ DTT, protease inhibitors). $10 \%$ of the lysate was retained as input control, $90 \%$ was used for the immunoprecipitation. Cell lysates were incubated with $20 \mu \mathrm{l}$ of Protein A/G agarose (Santa Cruz) pre-coupled with anti-CRM-1 (Novus Biologicals) in the presence (or absence) of recombinant His-RanQ69L protein (provided by $\mathrm{Dr}$ Virginie Gautier, UCD) and $20 \mathrm{mM}$ GTP $4{ }^{\circ} \mathrm{C}$ overnight. The immunoprecipitated protein complexes were washed thoroughly and then eluted by boiling in Laemmli sample buffer, before being subjected to SDS-PAGE and Western-Blot analysis.

\subsection{Immunofluorescent staining and confocal microscopy}

Hela cells were seeded on sterile glass cover slips in 6-well plates (and transfected with expression constructs where necessary). After $24 \mathrm{~h}$, they were washed, fixed with $4 \%$ paraformaldehyde, and permeabilised with $0.5 \%$ Triton-X-100. After blocking with BSA, cells were stained with the appropriate antibodies. Overexpressed DDX3 and DDX3 mutants were mostly HA-epitope tagged (only the K230E mutant is myc-epitope-tagged) and detected with antibodies against the epitope tag. For detection of the HA-tagged proteins, we either used a directly labelled anti-Ha antibody (anti-Ha-594, Invitrogen) or the same unlabelled anti-Ha primary antibody clone (16B12) (Covance) followed by Alexa-Fluor488- or AlexaFluor594-labelled anti-mouse secondary antibody (Invitrogen). Appropriate controls were included in the experimental set-up in order to rule out non-specific binding of antibodies and bleed-through of channels (e.g. secondary antibodies only, individual stainings as well as co-stainings). After staining, cells were mounted in SlowFade Gold Antifade Reagent containing DAPI (Invitrogen) on microscope slides and sealed using clear nail varnish. Cells were viewed using an Olympus Fluoview Confocal Microscope; and analysed using Olympus Fluoview FV10-ASW software. Multiple cells in several different frames were examined and recorded in each experiment, and experiments were repeated at least twice, most were repeated three or more times.

\subsection{High content analysis}

HeLa cells were grown in a 96wp and fixed and stained in the plate as described above. After secondary antibody incubation, DAPI (SigmaAldrich) was used at a concentration of $0.5 \mu \mathrm{g} / \mathrm{ml}$ to stain the nuclear DNA and Phalloidin-Tetramethylrhodamine B isothiocyanate (SigmaAldrich) was used at a concentration of $0.1 \mu \mathrm{M}$ to stain actin filaments. 
This was followed by further washing in PBS-Tween. Cells were then covered with $200 \mu \mathrm{l}$ PBS and analysed on a GE IN Cell Analyser 1000 (based in the Irish National Center for High Content Screening and Analysis, Trinity College Dublin). Data was collated by IN cell Software (GE Healthcare). DAPI and Phalloidin-staining were used to define nuclear and cytoplasmic regions, respectively, which were used as a mask for detection of changes in DDX3 staining. Each condition was measured in duplicate wells, with 10 frames captured per well, and between 1577 and 2401 cells analysed in total for each condition. The fraction of DDX3 in the nucleus was calculated by dividing backgroundcorrected nuclear intensity (IxA) by (background-corrected cytoplasmic intensity + background-corrected nuclear intensity).

\subsection{Cell cycle blocks}

HeLa cells were arrested at different cell cycle stages by treatment with different cell cycle inhibitors. A double thymidine block was used to arrest cells in early S-Phase: HeLa cells were plated at $25-30 \%$ confluency and grown in complete DMEM supplemented with $2 \mathrm{mM}$ thymidine for $18 \mathrm{~h}$. After this initial thymidine block, cells were washed in PBS twice and released into fresh complete DMEM for $9 \mathrm{~h}$. Cells were then added to complete DMEM supplemented with $2 \mathrm{mM}$ thymidine for $17 \mathrm{~h}$ for the second block. To arrest cells at G2/M boundary, a thymidine/nocodazole block was used: HeLa cells were plated at $40 \%$ confluency and grown in complete DMEM supplemented with $2 \mathrm{mM}$ thymidine for $24 \mathrm{~h}$. After initial thymidine block, cells were released into fresh complete DMEM for $3 \mathrm{~h}$. After release, $100 \mathrm{ng} / \mathrm{ml}$ nocodazole was added to cells for $12 \mathrm{~h}$. To arrest cells at the G0/G1 boundary, HeLa cells at $3-40 \%$ confluency were washed twice in PBS and grown in serum-free medium for $72 \mathrm{~h}$. Where required, cells were released from the different blocks into complete DMEM and harvested at specific time points to monitor cell cycle progression by Western Blot analysis.

\section{Results}

\subsection{DDX3's nuclear export is mediated by CRM1 and dependent on its $N$ - terminal conserved NES}

We first confirmed that DDX3's export is largely dependent on CRM1 , as previously observed by us and others. In HeLa cells, both endogenous and overexpressed DDX3 were predominantly cytoplasmic, but clearly accumulated in the nucleus after treatment with the specific CRM-1 inhibitor Leptomycin B (LMB) (Fig. 1A and B). The fact that DDX3 export is sensitive to LMB indicates that its export should be mediated by a classical NES, because LMB inhibits CRM-1 function by covalent modification of its NES-binding site (Kudo et al., 1998). As mentioned, there is controversial data about the functionality of the highly conserved N-terminal NES in different DDX3 orthologues. Thus, we wanted to clarify whether the N-terminal NES is required for export of human DDX3. We first demonstrated that a C-terminal deletion mutant of DDX3, containing only the first recA-like domain, specifically amino acids $1-408$, also accumulates in the nucleus following LMB treatment, confirming that its export still depends on CRM-1 despite partial removal of the proposed C-terminal CRM-1 binding site in DDX3 (Yedavalli et al., 2004) (Fig. 1C). Next, we generated a DDX3 mutant that does not contain the conserved N-terminal NES by truncating the first 22 amino acids of DDX3. This $\Delta 22$ mutant clearly accumulated in the nucleus in the absence of LMB treatment, suggesting it is not being exported efficiently (Fig. 1D). We also imaged localisation of additional $\mathrm{N}$-terminal truncation mutants, including 44-662 $(\Delta 44)$ (Supplementary Fig. 1a), 130-662 $(\Delta 130)$ and 139-662 ( $\Delta 139)$. All of these also accumulated in the nucleus as expected, and there was no additional effect compared to the $\Delta 22$ mutant (Fig. 1D). Interestingly though, the $\Delta 139-662$ mutant showed a more speckled distribution in the nucleus compared to both the $\Delta 130$ and $\Delta 22$ mutant.

Next, we mutated two of the conserved leucine residues in the NES,
L19 and L21, to alanines. This was sufficient to disrupt DDX3 export, as this NES mutant (mtNES) also clearly accumulated in the nucleus without LMB treatment (Fig. 1E). Finally, we affixed the 1-22 region of DDX3 containing the conserved NES back onto the $\Delta 130$ truncation mutant of DDX3, which prior to this manipulation accumulated in the nucleus like other N-terminal truncation mutants (see Fig. 1D). Addition of the NES largely restored localization of the $\Delta 130$ truncation mutant to the cytoplasm and also conferred sensitivity to LMB treatment (Fig. 1F). Similar effects were also seen when we affixed the NES to the $\Delta 44$ and $\Delta 139$ truncation mutants (Supplementary Fig. 1a). In addition, the NES (1-22 region of DDX3) was able to enhance export of an unrelated protein (the transcription factor ZNF512) when we fused it to its N-terminus (Supplementary Fig. 1b). These results clearly confirm that the conserved leucine-rich region between aa 1 and 22 in DDX3 contains a functional NES.

Finally, we tested whether enzymatic activity of DDX3 is required for nuclear export, because an ATPase-deficient mutant of its orthologue An3 mutant was shown to accumulate in the nucleus (Askjaer et al., 2000). However, the K230E mutant of human DDX3, which has no ATPase or RNA unwinding activity, behaved like wild-type DDX3 in our shuttling assays. It was largely cytoplasmic when expressed in cells, and accumulated in the nucleus only after treatment with LMB (Fig. 1G), thus suggesting that enzymatic activity is not required for nuclear export of human DDX3.

Taken together, these experiments clearly demonstrate that the conserved N-terminal NES and CRM-1 are required for efficient export of human DDX3 from the nucleus, whereas its enzymatic activity is not.

\subsection{DDX3 binding to CRM-1 is mediated by the N-terminal NES}

To test whether the physical interaction between DDX3 and CRM-1 also depends on the N-terminal NES, we tried to co-immunoprecipitate DDX3 or DDX3 mutants lacking either the N-terminal NES and/or the Cterminal region with endogenous CRM-1. To this end, we transfected cells with expression plasmids for HA-tagged wild-type DDX3, fulllength DDX3 containing the two leucine substitutions in the NES (mtNES), a C-terminally truncated mutant (1-408), or an N- and Cterminal deletion mutant (139-408). We then immunoprecipitated endogenous CRM-1 from the cell lysates in the presence of recombinant non-hydrolysable His-RanQ69L-GTP, which stabilizes CRM-1-cargo interactions (Guttler et al., 2010). Under these conditions, wild-type DDX3 and 1-408 DDX3 co-immunoprecipitated with CRM-1, while the two DDX3 mutants lacking a functional N-terminal NES did not (mtNES, 139-408) (Fig. 2). This suggests that DDX3 behaves like a classical CRM-1 cargo protein, with both binding to CRM-1 and export from the nucleus being dependent on the conserved leucine-rich NES sequence in the $\mathrm{N}$-terminus.

\subsection{DDX3 contains at least two independent Nuclear Localization Signals (NLS) in the $N$-terminal region}

The cellular localization of DDX3 is influenced by the balance of import and export, thus it is also important to understand how DDX3 is imported into the nucleus. However, no information is available to date about the determinants of DDX3's nuclear import. From our experiments studying DDX3's nuclear export described above, we are also able to draw some conclusions regarding DDX3's import into the nucleus, seeing as mutants that accumulate in the nucleus when export is inhibited have to be imported in the first place. DDX3's import therefore also does not require its enzymatic activity (Fig. 1G), and deletion of the N-terminus or C-terminus do not prevent import (mutants 139-662 and 1-408 were imported into the nucleus) (Fig. 1D and C). Nuclear localization signals (NLSs) are harder to identify than export signals, as there are different importin proteins and their recognition sequences are more variable. We used several NLS prediction software programmes to identify putative NLSs in DDX3. The software PSORT II 
A

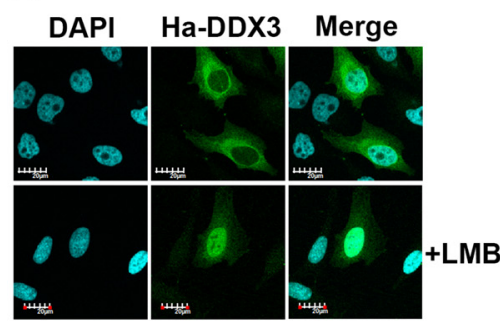

D

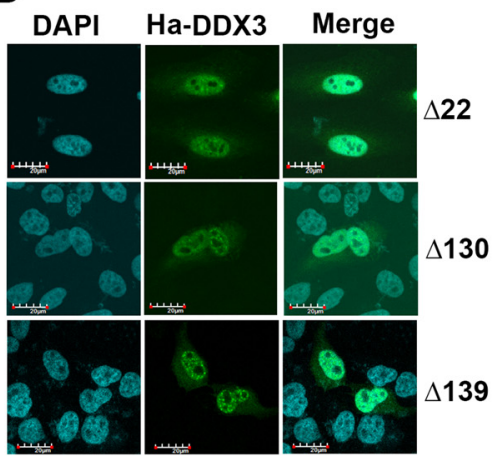

B

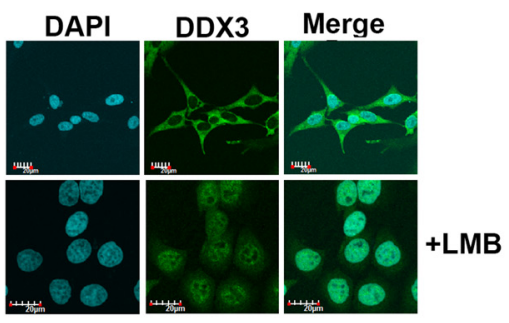

E

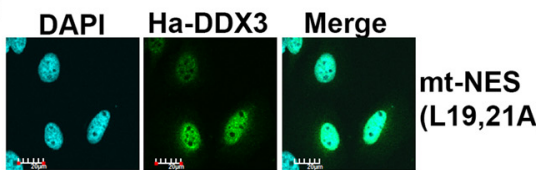

$\mathbf{F}$
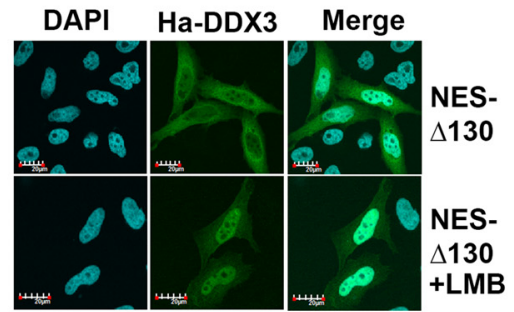

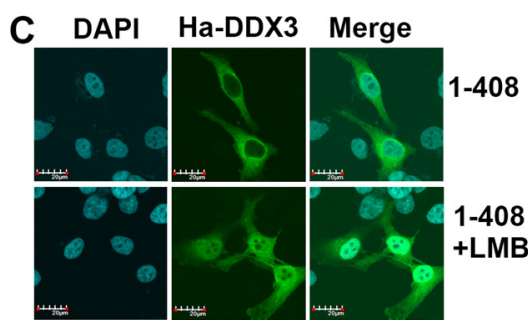

G

DAPI myc-DDX3 Merge

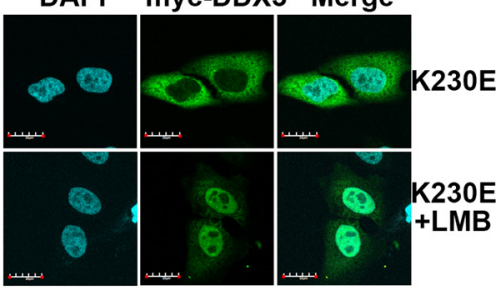

$\mathrm{H}$

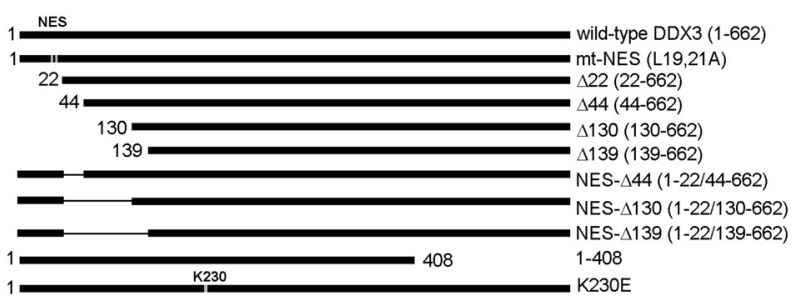

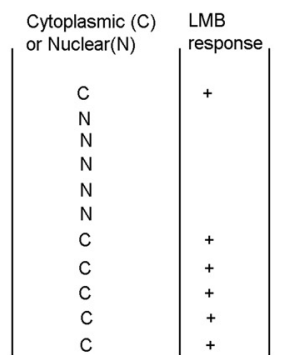

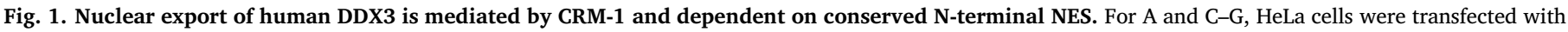

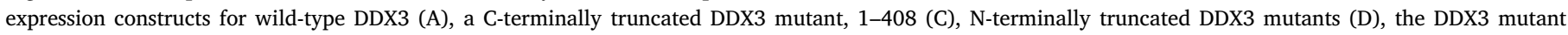

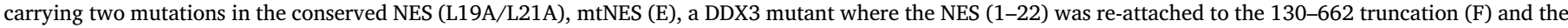

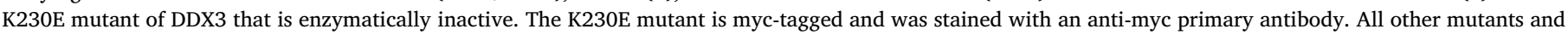

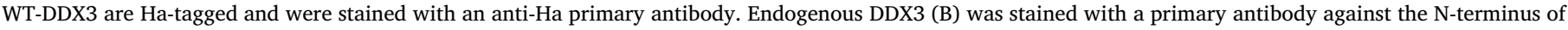

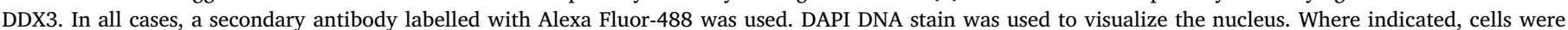

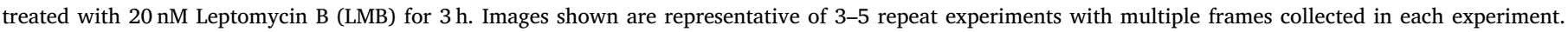

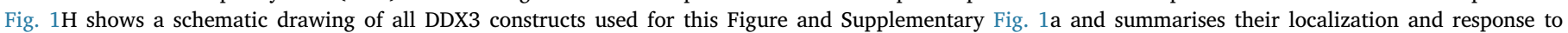
Leptomycin B (LMB, + indicates that mutant accumulates in nucleus following LMB treatment).

can identify classical NLS sequences in proteins (Nakai and Horton, 1999), and suggested a putative classical NLS between amino acids 211 and 219 of DDX3, thus located within the first recA-like domain. Using site directed mutagenesis we changed two crucial positively charged residues to alanines, namely the lysines at positions 215 and 217 (Fig. 3A). This 'NLS1' mutation was generated in the context of full length DDX3 (NLS1- 1-662) and in the C-terminal truncation 1-408 (NLS1-1-408). We then transfected HeLa cells with expression

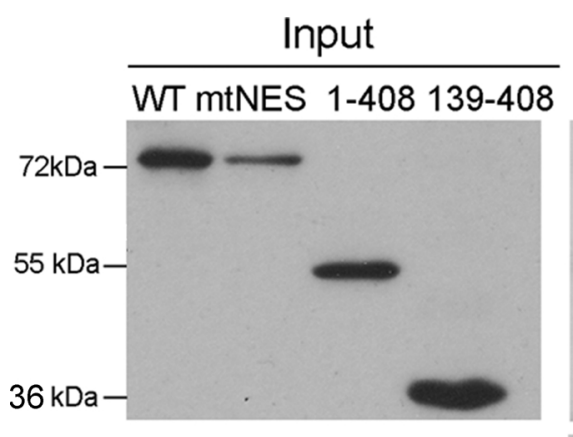

$90 \mathrm{kDa}-$
IP: CRM-1
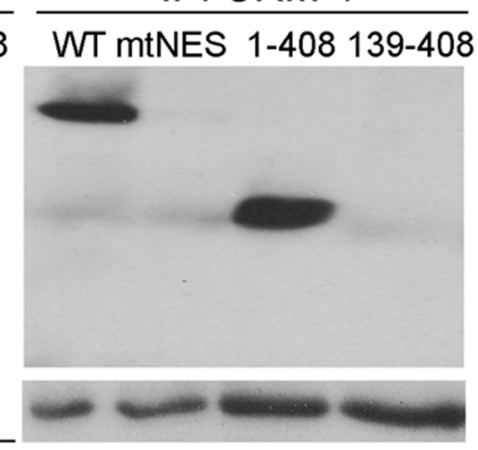

WB: $\mathrm{Ha}$

WB: CRM-1

Fig. 2. An intact N-terminal NES is required for DDX3 binding to CRM-1. HEK293T cells were transiently transfected with expression constructs for Ha-tagged wild-type (WT) DDX3, for full-length DDX3 carrying the two point mutations in the NES (mtNES), for the $1-408$ or the 139-408 truncation mutants. Endogenous CRM-1 was then immunoprecipitated from cell lysates in the presence of recombinant non-hydrolysable HisRanQ69L-GTP, which stabilizes CRM-1 cargo interactions. Samples were then subjected to SDS-PAGE and Western Blot analysis with antibodies against the Ha epitope tag (detecting DDX3) and CRM-1. Shown is one representative experiment out of two. 
A

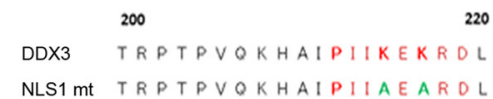

C 255 $\begin{array}{ll}\text { DDX3 } & \text { KENGRYGRRKQYPISLVLAPT } \\ \text { NLS2 mt } & \text { KENGAAGAAKQYPISLVLAPT }\end{array}$

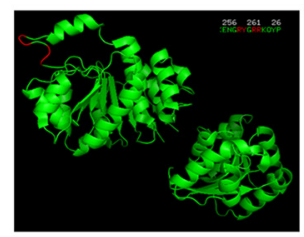

E

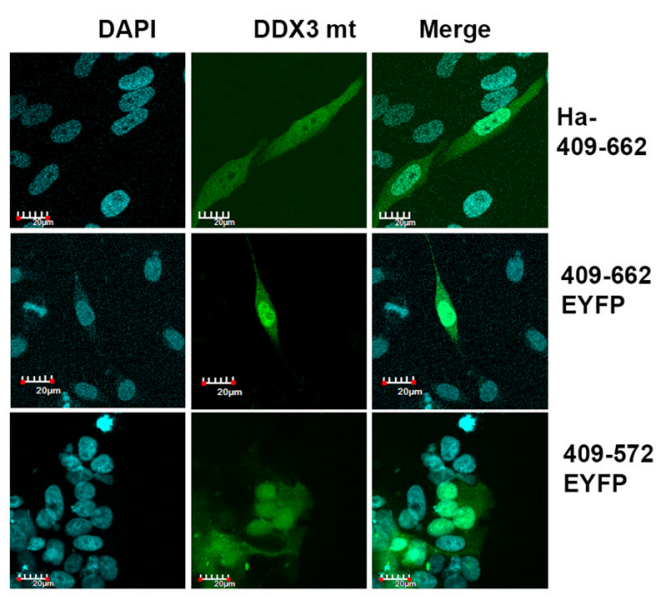

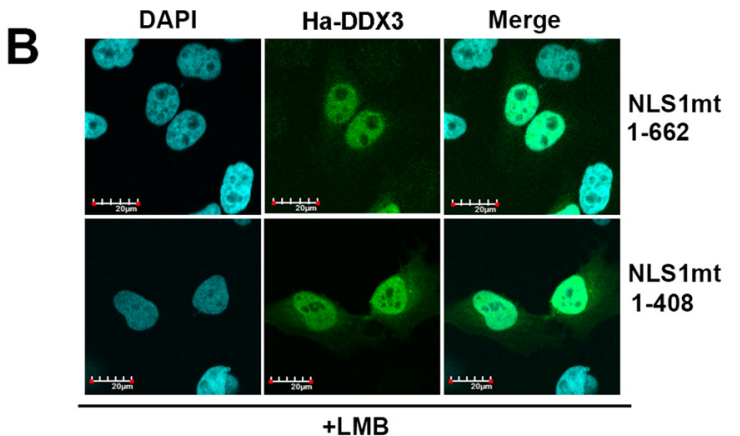

D

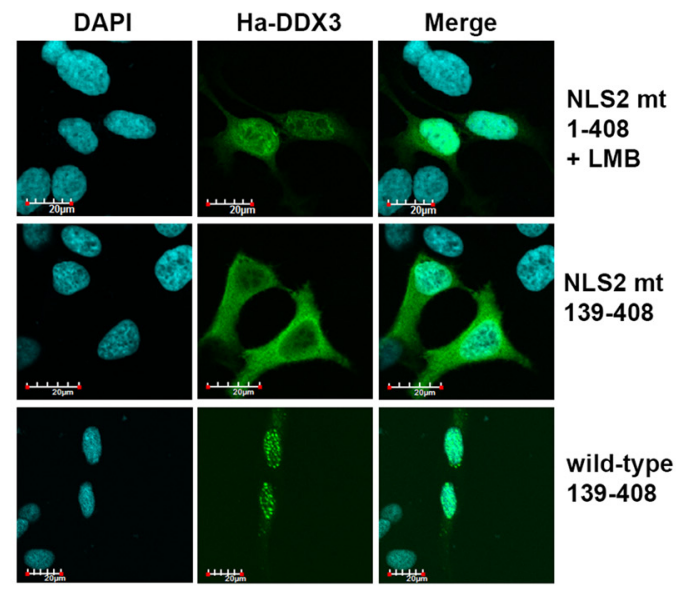

$\mathrm{F}$

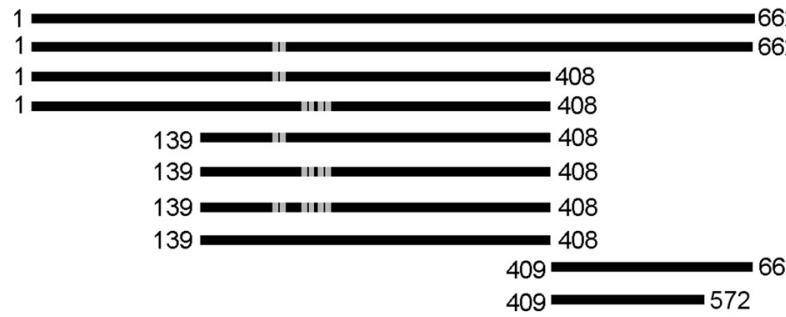

662 DDX3 (wild-type)

2 NLS1 1-662 (K215,217A)

NLS1 $1-408$

NLS2 1-408 (R259,Y260,R262,R263)

NLS1 139-408

NLS2 139-408

NLS1+2 139-408

139-408 (wild-type)

2 409-662 (also 409-662 EYFP) 409-572 (EYFP)

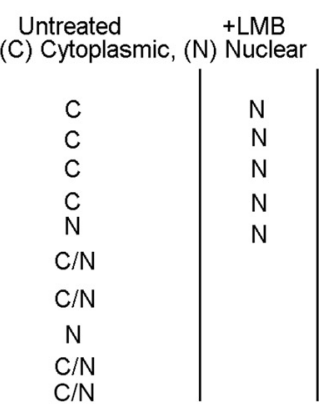

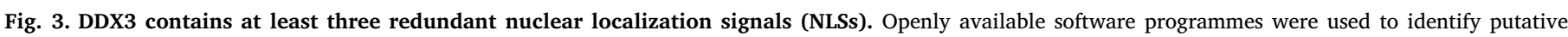

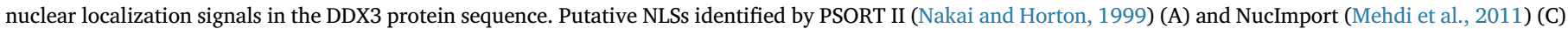

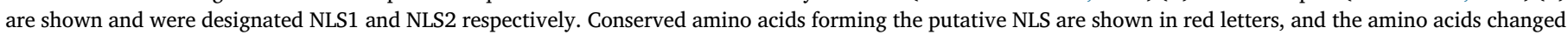

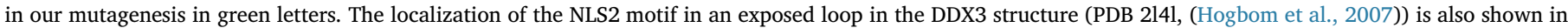

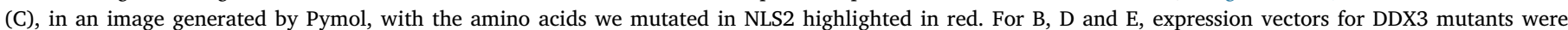

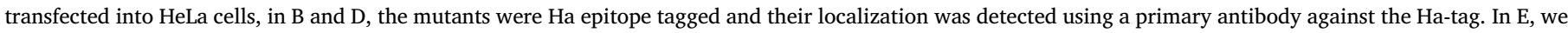

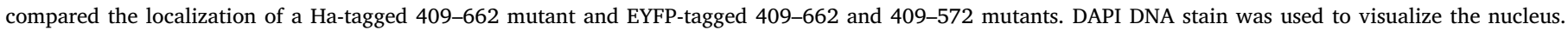

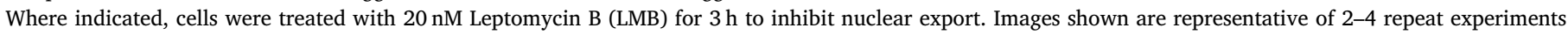

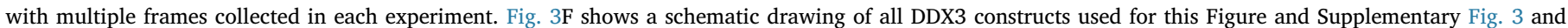

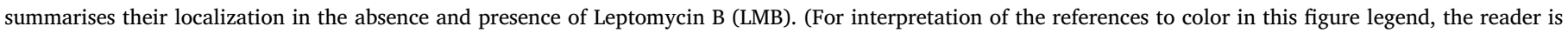
referred to the web version of this article).

constructs for these NLS1 mutants, and treated the transfected cells with LMB to inhibit nuclear export. Both the long and short NLS1 mutant were cytoplasmic in untreated cells as expected (not shown) and nuclear in LMB-treated cells (Fig. 3B), indicating that import was not affected by the mutations. This either suggests that the identified putative NLS at position 211-219 is not functional, or that even the Nterminal 1-408 fragment of DDX3 contains more than one redundant
NLS. We next used the software NucImport to identify further putative NLSs, as it was suggested to identify NLSs better than previously published software and is not restricted to classical NLSs (Mehdi et al., 2011). NucImport identified a putative NLS at amino acids 259-264, located in an exposed loop in the protein structure of DDX3 (Fig. 3C). Mutagenesis of this putative NLS was carried out in the 1-408 truncated DDX3, with a total of four amino acids (R259, Y260, R262, R263) 
A

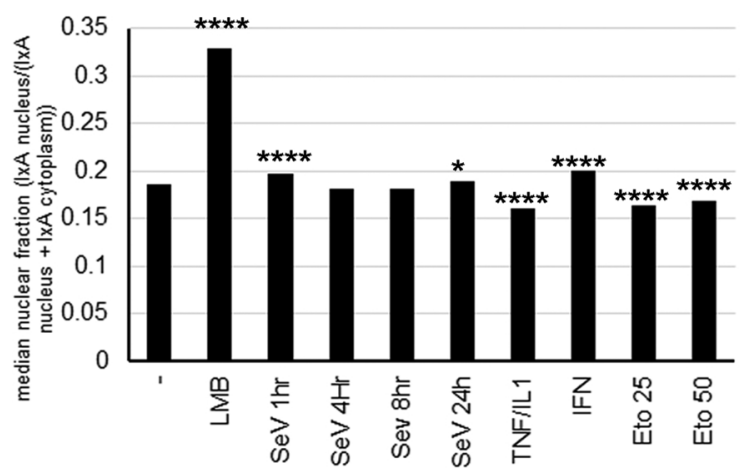

B

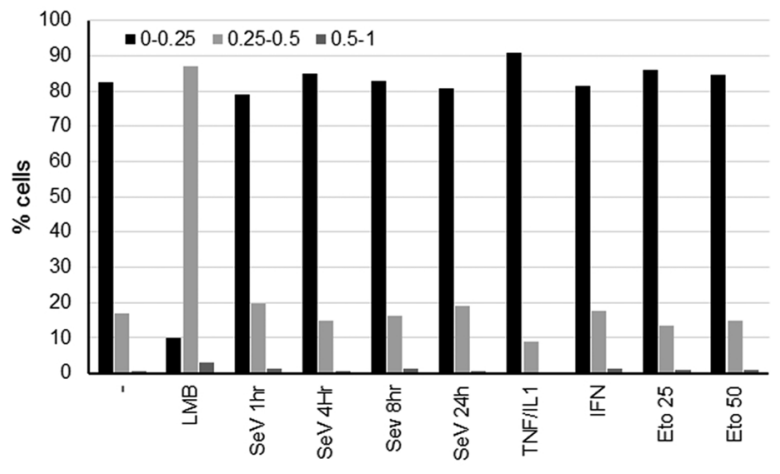

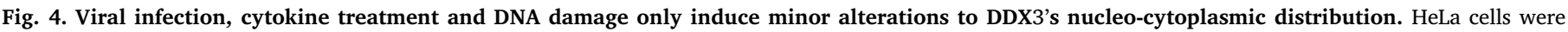

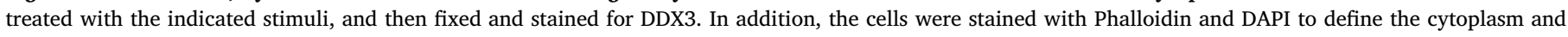

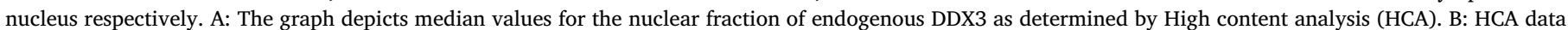

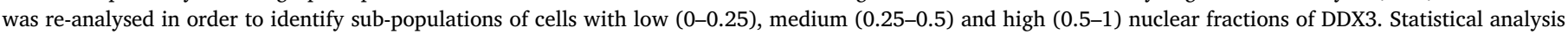

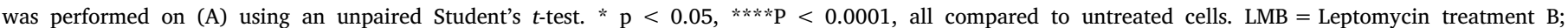

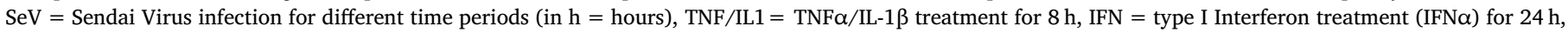
Eto $=$ Etoposide treatment at 25 or $50 \mu \mathrm{M}$.

changed to alanines, and the resulting mutant was called 'NLS2' (Fig. 3C). HeLa cells overexpressing HA-tagged NLS2 (1-408) were treated with LMB to inhibit export. The NLS2 mutant was also cytoplasmic in untreated cells (not shown) and nuclear in cells treated with LMB (Fig. 3D), suggesting that import was again not disrupted by mutation of this second putative NLS. However, we next decided to truncate the N-terminus in the NLS2 mutant and generated the '139-408 NLS2' mutant. The wild-type 139-408 truncation of DDX3 localises to the nucleus in the absence of LMB treatment due to loss of the NES, and shows a speckled distribution in the nucleus (Fig. 3D, bottom row), similar to the 139-662 mutant (Fig. 1D). Interestingly, the 139-408 truncation carrying the NLS2 point mutations (Fig. 3D, middle row) was markedly more cytoplasmic than either the 1-408 NLS2 mutant (in the presence of LMB, top row) or the wild-type 138-408 truncation (bottom row). This comparison suggested that there is an additional NLS present in the N-terminal region (aa 1-139) that is able to mediate import of the 1-408 construct in the absence of NLS2. NLS2 on the other hand is functional in the absence of the N-terminal NLS, as it mediates import of the 139-408 truncation. We also further confirmed that the putative NLS1 investigated in Fig. 3A and B does not contribute to import of the 139-408 truncation, by generating the corresponding NLS1 139-408 mutant and the NLS1 + 2 139-408 double mutant. The NLS1 139-408 mutant accumulated in the nucleus similar to wild-type 139-408, and there was also no significant difference between NLS2 139-408 and the NLS1 + 1 139-408 double mutant (Supplementary Fig. 3).

In conclusion, we have identified two functional NLSs in DDX3, one in the N-terminal extension and one in the first recA-like domain, while the only putative NLS identified by PSORT II appears to be non-functional.

\subsection{The C-terminal RecA-like domain of DDX3 is also independently imported into the nucleus}

We also observed nuclear localization of a truncation mutant comprising only the C-terminal part of DDX3, aa 409-662, which includes the second recA-like domain and the flexible C-terminal extension, but contains neither of the NLSs identified above (Fig. 3E). However, because the distribution of the 409-662 truncation mutant was quite diffuse across both cytoplasm and nucleus, and because it is only approximately $30 \mathrm{kDa}$ large, we considered that it might simply diffuse into the nucleus passively. We thus increased the size of the truncation mutant by sub-cloning it into a vector generating a fusion protein with Enhanced Yellow Fluorescent Protein (EYFP). EYFP is approximately $27 \mathrm{kDa}$, therefore the resulting fusion protein is around $57 \mathrm{kDa}$ and too large to passively diffuse through the nuclear pore. We found that 409-662-EYFP and 409-572-EYFP mutants also localised to the nucleus, maybe even more clearly than the Ha-tagged 409-662 protein (Fig. 3E), suggesting that nuclear import is indeed facilitated independently for the C-terminal domain of DDX3. The 409-572 truncation corresponds roughly to the second recA-like domain. None of the NLS prediction software programmes identified a putative NLS in this region, hence we were unable to further narrow down the sequence responsible for mediating its import.

In summary, we demonstrated that DDX3 contains at least three independent NLSs: one in the flexible N-terminus (N-NLS) (aa 1-139), one between aa 259-263 in an exposed surface loop extending from the first RecA like domain (NLS2), and one in the C-terminal RecA-like domain between aa 409-572.

\subsection{Can the subcellular localization of DDX3 be altered?}

We were also interested in the question whether DDX3's distribution between the cytoplasm and the nucleus can be altered by cellular conditions. In order to more objectively and quantitatively analyse the subcellular distribution of DDX3, we used a High Content Analyser to determine the nuclear/cytoplasmic ratio. To this end, we stained DDX3 in HeLa cells as usual, but combined it with a Phalloidin F-actin stain (to define the cytoplasmic area) and the DAPI DNA stain (to define the nucleus). The intensity of nuclear and cytoplasmic DDX3 staining was determined for all cells in ten different fields per well (and two wells/ condition). This resulted in approximately 2000 cells being analysed for each condition. For endogenous DDX3 in untreated HeLa cells, the median value for the nuclear fraction ((IxA nucleus)/(IxA nucleus) + (IxA cytoplasm)) was 0.185. As a control for our set-up, we treated cells with LMB to inhibit nuclear export of DDX3. This clearly increased the median value for the nuclear fraction to 0.329 (unpaired Student's $T$-test, $\mathrm{p}=0$ ) (Fig. 4A). We also calculated the percentages of cells that fell into the $0-0.25$, the $0.25-0.5$ and the $0.5-1$ range (with 1 representing fully nuclear DDX3). For untreated HeLa cells, $82.36 \%$ of cells were in the lowest range of $0-0.25,16.91 \%$ of cells in the $0.25-0.5$ range, and $0.66 \%$ of cells in the highest range of $0.5-1$. In contrast, for LMB-treated HeLa cells, only $10.06 \%$ of cells were in the lowest range, whereas $86.93 \%$ were in the $0.25-0.5$ range and $3 \%$ in the $0.5-1$ range 
(Fig. 4B). Thus, as expected, a clear shift towards increased localization of DDX3 was observed with LMB treatment. We then used this system to test the effects of a viral infection (Sendai Virus, SeV), cytokine stimulation (TNF $\alpha / \mathrm{IL} 1 \beta$ or type I interferon, IFN)) and DNA damage (induced by etoposide treatment) on the nucleo-cytoplasmic distribution of DDX3. The median values for the nuclear fraction of DDX3 appeared to remain largely unchanged for these treatments (Fig. 4A). However, some of the small changes were statistically significant, including small increases in DDX3's nuclear fraction for SeV infection at $1 \mathrm{~h}(0.198, \mathrm{p}=1.8 \mathrm{E}-09)$ and $24 \mathrm{~h}(0.2, \mathrm{p}=0.02)$ and IFN treatment $(0.21, p=1.67 \mathrm{E}-8)$. For these conditions, there was also a corresponding small increase in the percentage of cells in the $0.25-0.5$ range (e.g. $19.75 \%$ for $1 \mathrm{~h} \mathrm{SeV}$ infected cells compared to $16.91 \%$ in the uninfected cells) and the $0.5-1$ range (1.4\% compared to $0.66 \%$ ) (Fig. 4B). For TNF/IL-1 stimulation, there was a statistically significant small decrease in the median value for the nuclear fraction of DDX3 $(0.172$, $\mathrm{p}=1.16 \mathrm{E}-21$ ). Etoposide treatment decreased the median value for the nuclear fraction very slightly to $0.182(25 \mathrm{u} \mathrm{M}, \mathrm{p}=5.75 \mathrm{E}-05))$ and 0.186 ( $50 \mathrm{uM}, \mathrm{p}=1.22 \mathrm{E}-08$ ), but conversely there appeared to be a slight increase in the percentage of cells in the highest 0.5-1 range ( $0.83 \%$ for $50 \mu \mathrm{M}$ Etoposide compared to $0.66 \%$ in untreated cells). Despite the statistical significance of the data, it is questionable whether such small shifts in DDX3's nucleo-cytoplasmic distribution result in meaningful changes to cellular behaviour.

\subsection{DDX3's subcellular localization is affected by cell cycle stage}

Finally, we decided to investigate whether the cell cycle stage affects DDX3's subcellular localization. To this end, we identified cells at different stages of the cell cycle on our stained slides. They were identified based on the shape of the cell and chromatin condensation level, and by co-staining with $\alpha$-Tubulin for visualization of the mitotic spindle or phospho-Histone $\mathrm{H} 3$ antibody, which detects endogenous levels of histone $\mathrm{H} 3$ only when phosphorylated at Ser28. This phosphorylation is tightly correlated with chromosome condensation during mitosis and meiosis, and can therefore be used as a marker for mitotic cells.

We first stained untreated 'unsynchronised' HeLa cells and identified mitotic cells based on the criteria outlined above. For example, prophase/prometaphase cells were identified based on phospho-H3 staining and an intact rounded nucleus. Levels of nuclear DDX3 appeared to be increased slightly in these cells (Fig. 5A, white arrows). We also observed increased DDX3 staining around the aligned condensed chromosomes during metaphase and anaphase (Fig. 5A, white arrowheads). We quantified nuclear localisation of DDX3 by determining the Pearson's Correlation Coefficient (PCC) between Alexa-Fluor488 staining (DDX3) and the DAPI nuclear stain for non-mitotic cells (i.e. those not staining for phospho-H3) and mitotic cells (pro/prometaphase and metaphase cells based on shape of nucleus). While the PCC was negative for non-mitotic cells, it was 0.42 and significantly increased for pro/prometaphase cells ( $\mathrm{p}=1.25 \mathrm{E}-5$, unpaired $t$-test). Similar observations were made when DDX3 was co-stained with $\alpha$-tubulin to visualize the mitotic spindle (Fig. 5B). Again, DDX3 appeared to be more nuclear during pro/prometaphase (white arrows), and to co-localise to some extent with the mitotic spindle (tubulin) during metaphase through to telophase/cytokinesis (white arrowheads) (Fig. 5B). To further confirm these observations, we treated HeLa cells with cell cycle inhibitors to arrest them at different mitotic stages. Cells were arrested at the G0/G1 boundary with serum starvation, in early S-Phase with a double thymidine block, or at the G2/M boundary with nocodazole, followed by staining and imaging of endogenous DDX3. HeLa cells blocked at the G0/G1 or in S-Phase had cytoplasmic DDX3 (Fig. 5C and D). HeLa cells blocked at the G2/M boundary with nocodazole however had increased nuclear DDX3 (Fig. 5E). Nocodazole causes cells to arrest in prometaphase, suggesting that DDX3 becomes more nuclear during prophase. This is also consistent with our imaging of unarrested
HeLa cells, where DDX3 appeared to be more nuclear in cells identified to be in prophase/prometaphase by Histone $\mathrm{H} 3$ staining and the morphology of the nucleus and the cell (Fig. 5A).

\subsection{Phosphorylation of DDX3 at threonine 204 and 323 does not regulate its nuclear accumulation}

DDX3 has been shown to be phosphorylated by Cyclin B at threonines 204 and 323 during mitosis (Sekiguchi et al., 2007). Cyclin B levels rise during early mitosis, and we thus hypothesized that phosphorylation of DDX3 by Cyclin B could mediate its observed nuclear accumulation during prophase. To investigate this, we first tested an antibody that is supposedly specific for DDX3 phosphorylated at Thr323 available from Abcam (ab61043). In immunofluorescent staining, this antibody produced a nuclear staining (which co-localised with staining for a nuclear speckle marker) (data not shown), seemingly supporting our hypothesis that DDX3 phosphorylation might lead to its nuclear accumulation. However, when we tested the antibody in Western Blots, it failed to produce a band at the correct size for DDX3 $(73 \mathrm{kDa})$ and instead produced a band at $130 \mathrm{kDa}$ (data not shown). We thus suspect that this antibody cross-reacts with a $130 \mathrm{kDa}$ protein present in nuclear speckles, and is not suitable for specifically detecting phosphorylated DDX3. We then generated mutants of DDX3 where we substituted the threonines at positions 204 and 323 with either phospho-deficient alanines (TA2 mutant) or phospho-mimetic aspartic acid (TD2 mutant). HeLa cells were transfected with expression constructs of these DDX3 mutants and stained as previously described (for the Ha-epitope tag). The phospho-deficient TA2 (T204A,T323A) and the phosphomimetic TD2 (T204D,T323D) double mutants localised predominantly to the cytoplasm (Fig. 6), suggesting that phosphorylation at these threonine residues is not driving nuclear accumulation of DDX3. Import of DDX3 was also not affected by these mutations, as they accumulated in the nucleus following treatment with LMB (Fig. 6).

In conclusion, while we provide evidence that DDX3 becomes more nuclear during early mitosis (prophase and prometaphase) and might stay associated with the mitotic spindle during chromosome segregation, we have been unable to identify the mechanism behind this change in localization, having ruled out phosphorylation at threonines 204 and 323 as the cause.

\subsection{DDX3 expression is upregulated during mitosis}

We also wanted to determine whether protein levels of DDX3 are altered during the cell cycle. First, we again used serum starvation, double thymidine or nocodazole treatment to arrest Hela cells in G0/ G1, early S-Phase, or G2/M respectively, followed by western blot detection of endogenous DDX3. Compared to untreated cells, HeLa cells blocked at G0/G1 and in S-Phase had reduced levels of DDX3, while cells blocked at G2/M with nocodazole had clearly increased levels of DDX3 (Fig. 7A). We next decided to monitor changes in DDX3 expression more closely during cell cycle progression. To this end, HeLa cells were blocked in early S-Phase using a double thymidine block, and then released into growth medium. We took samples for western blot analysis for $18 \mathrm{~h}$ after the release, and probed also for Cyclin E1 (highly expressed during G1), Cyclin A (highly expressed during S and G2) and Cyclin B (highly expressed during early M phase). Cyclin A was detected from $0 \mathrm{~h}$ to $15 \mathrm{~h}$ after release with the strongest expression, which should occur in G2 phase, being detected $6 \mathrm{~h}$ after release. Cyclin $\mathrm{B}$ expression is expected to peak in early mitosis (M-phase) and then to strongly decrease. We observed strong Cyclin B expression 6 and $9 \mathrm{~h}$ after release, and its degradation had occurred by $12 \mathrm{~h}$ after release. DDX3 levels were clearly increased $6 \mathrm{~h}$ and $9 \mathrm{~h}$ after release, before decreasing again $12 \mathrm{~h}$ after release. The increased DDX3 levels therefore correlated with the strongest expression of mitotic cyclin B, confirming that DDX3 levels are increased in G2 and early M-phase. This is in agreement with the observation made in Fig. 7A, where cells arrested at 
A

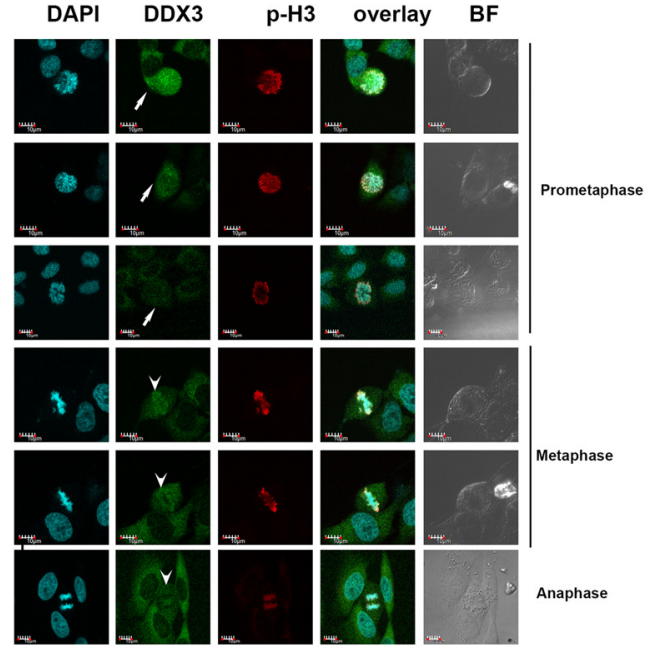

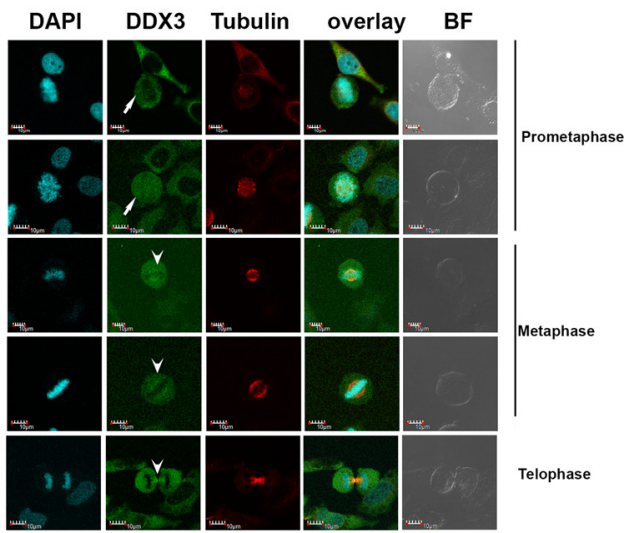

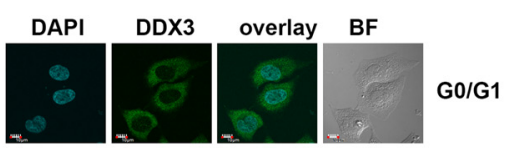

D

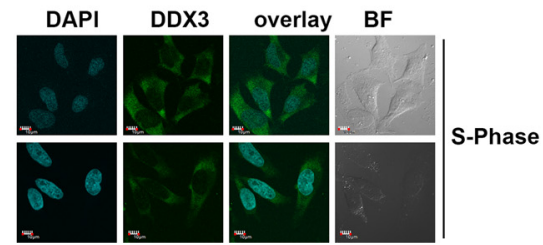

E

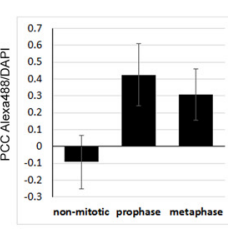

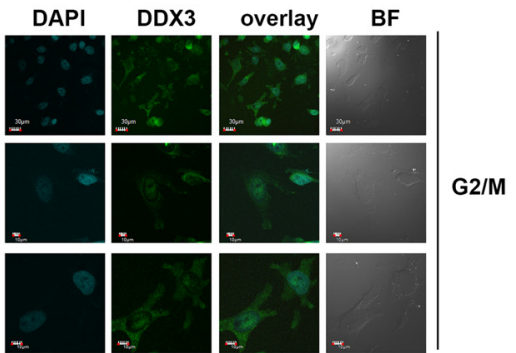

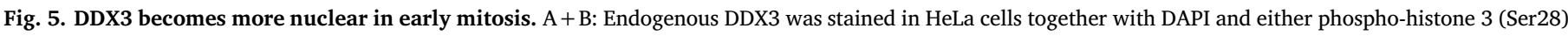

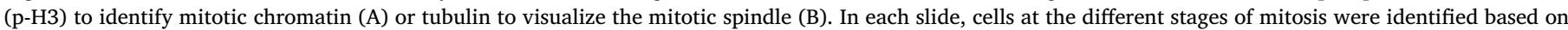

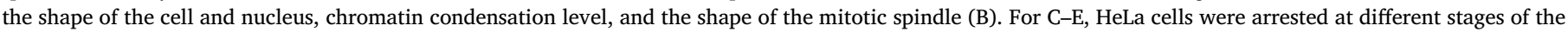

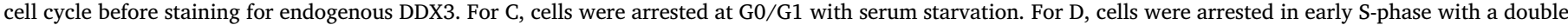

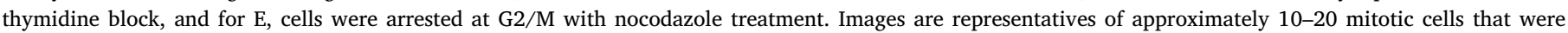

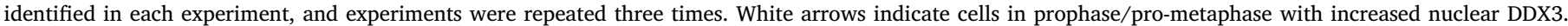

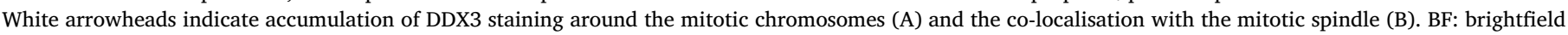
image.

\section{G2/M showed increased DDX3 expression.}

In summary, we have demonstrated that DDX3's expression and subcellular localisation are regulated during the cell cycle. DDX3 protein expression increased in early M-phase (prophase/prometaphase), also correlating with the cell cycle stage where its localization became more nuclear. This further supports an important role for DDX3 during mitosis, such as its proposed role during chromosome segregation (Chen et al., 2017; Pek and Kai, 2011).

\section{Discussion}

Our study identified NLS and NES sequences in human DDX3 and investigated the regulation of its nucleo-cytoplasmic shuttling, with the aim of identifying factors that could lead to an alteration in its subcellular distribution.

\subsection{Nuclear export of DDX3 is mediated by the $N$-terminal conserved NES}

Our data clearly demonstrates that the conserved N-terminal NES is required for export of human DDX3 from the nucleus in HeLa (Fig. 1) and also HEK293 cells (not shown). Recent publications by Frohlich et al. (2016) and Heerma van Voss et al. (2017b) share this assessment. In co-immunoprecipitation experiments that we carried out in the presence of non-hydrolysable Ran-GTP, DDX3 binding to endogenous CRM-1 was also dependent on the N-terminal NES, as would be expected for classical CRM-1 cargo. However, Yedavalli et al (2004) had previously shown that DDX3 binds to CRM-1 through a C-terminal region (311-507) and in a Ran-GTP-independent manner. It is not fully clear to us what causes the discrepancy between these two findings. We did detect weaker non-discriminatory binding of all DDX3 mutants to CRM-1 in the absence of Ran-GTP (see expanded blots for CRM-1 IP shown in Supplementary Fig. 2). However, in the presence of Ran-GTP, which mimics conditions present in the nucleus, CRM-1 exclusively bound NES-containing DDX3 constructs (Fig. 2 and Supplementary Fig. 2). We are not sure whether non-NES mediated binding of DDX3 to CRM-1 in the absence of Ran-GTP has a physiological function or is caused by an experimental artefact.

As a side note, we usually saw exclusion of DDX3 from nucleoli when nuclear accumulation of DDX3 was achieved by truncating the NES or treating cells with LMB (e.g. Figs. 1 and 6). This is different to the observations made by van Voss et al. (Heerma van Voss et al., 2017b), who observed increased DDX3 staining in the nucleoli compared to the nucleoplasm in their recent study. It is unclear what causes these different staining patterns as both studies were performed in HeLa cells, but it raises the interesting question whether DDX3 distribution between nucleoli and nucleoplasm could also fluctuate depending on 


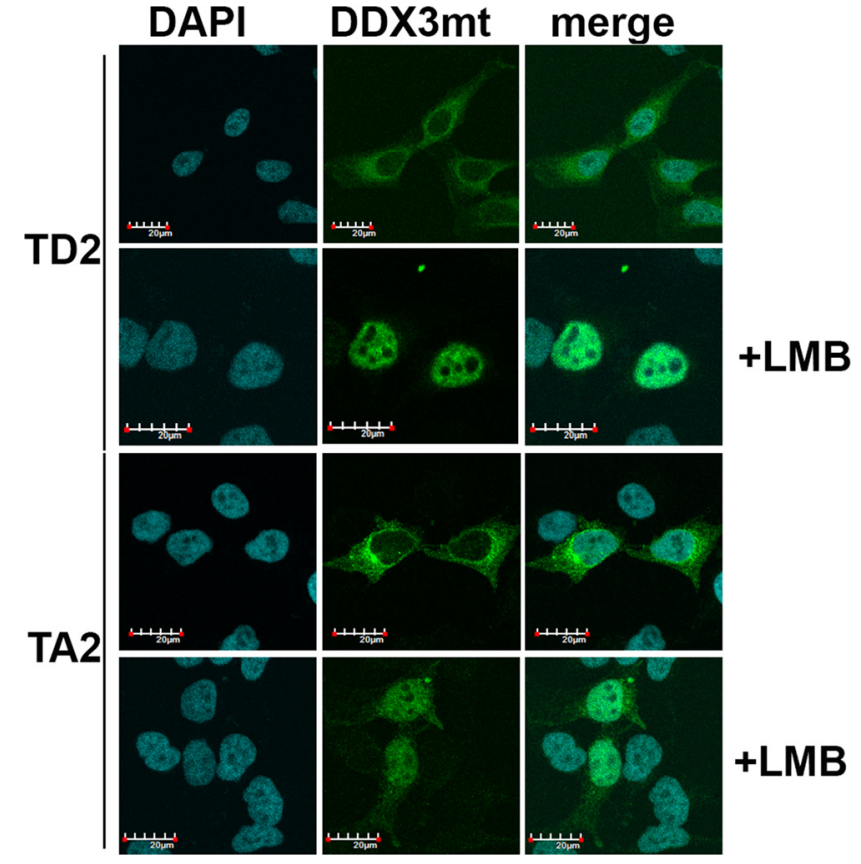

Fig. 6. Mutation of putative Cyclin B phosphorylation sites in DDX3 does not affect import or export of DDX3. Threonine 204 and 323, which have been described as phosphorylation sites for Cyclin B, were mutated to either alanine (TA2) or aspartic acid (TD2) to generate phospho-deficient and phosphor-mimetic mutants respectively. Expression vectors for the two Ha-tagged DDX3 mutants (DDX3mt) were then transfected into HeLa cells, and localization of the DDX3 mutants visualized by staining with an antibody for the Haepitope tag, followed by an Alexa Fluor488-labelled secondary antibody. DAPI DNA stain was used to visualize the nucleus. Where indicated, cells were treated with $20 \mathrm{nM}$ Leptomycin B (LMB) for $3 \mathrm{~h}$. Images shown are representative of 2 repeat experiments with multiple frames collected in each experiment.

unidentified cellular conditions. That DDX3 can traffic into different nuclear compartments is also suggested by the distinct staining pattern we observed for the N-terminally truncated DDX3 mutants 139-662 and 139-408, which resembled nuclear speckles (Figs. 1D and 3 D) (Spector and Lamond, 2011). Thus, it is possible that DDX3 is pulled into nucleoli or nuclear speckles depending on cellular conditions, e.g. cellular stress or an increased requirement for ribosome biogenesis (Boulon et al., 2010).

\subsection{Multiple nuclear localization signals exist within the DDX3 sequence}

We were also keen to identify the nuclear localization signals (NLS) in DDX3, as no information is available to date about the regulation of DDX3's nuclear import. However, NLSs are harder to identify than NESs, and several NLS prediction software programmes failed to identify strong hits within the DDX3 sequence. The putative classical NLS identified by PSORT II (Nakai and Horton, 1999) between aa 211-219 ('NLS1') appears to be non-functional, as its mutation did not prevent import of DDX3, even of the 139-408 truncated protein (Supplementary Fig. 3). 'NLS1' is also still present in the 139-408-truncated 'NLS2' mutant, which did not accumulate in the nucleus (Fig. 3D). Thus it is unable to mediate import in the absence of 'N-NLS' and 'NLS2'. NucImport (Mehdi et al., 2011) identified 'NLS2', a putative NLS between aa 259-264, which is located in an exposed loop according to the DDX3 crystal structure. Mutation of this sequence alone also did not prevent import of full-length DDX3 or the 1-408 truncation. However, additional truncation of the $\mathrm{N}$-terminal tail largely prevented import (Fig. 3D). This suggests that 'NLS2' (259-264) is functional, but that an additional import signal is present between amino acids 1 and 139 of DDX3 (a putative 'N-NLS'), which is likely responsible for export of the 'NLS2'-mutated 1-408 fragment. We also observed independent nuclear import of the second recA-like domain of DDX3 (aa 409-572), which contains neither 'NLS2' nor 'N-NLS'. None of the NLS prediction programmes returned any hits for this region of DDX3, so we were unable to further narrow done the sequence(s) in DDX3 that are responsible for import of the C-terminal recA-like domain. The lack of putative NLSs in this region of DDX3 might also indicate that it can be imported in an unconventional manner, e.g. via Transportin in a PY-NLS independent manner, via the Calmodulin pathway or perhaps "piggy backing" into the nucleus with another protein (Wagstaff and Jans, 2009). In any case, there clearly is redundancy in the NLSs present in DDX3, with us having identified at least three regions in DDX3 that can independently mediate its nuclear import.

\subsection{Regulation of DDX3's subcellular localization}

With DDX3 inhibitors being developed as potential anti-cancer and anti-viral drugs (Bol et al., 2015; Heerma van Voss et al., 2017a; Heerma van Voss et al., 2018; Maga et al., 2011; Radi et al., 2012), it is important to understand in detail how DDX3 contributes to different cellular functions, especially those related to cellular growth, proliferation, and migration, and genome integrity (Sharma and Jankowsky, 2014). For this study, we investigated the nuclear-cytoplasmic shuttling of DDX3. Given the distinct cytoplasmic and nuclear functions of DDX3, it is tempting to speculate that an alteration in the nuclear-cytoplasmic distribution of DDX3 could affect its participation in various cellular processes with downstream consequences for cellular phenotypes. Indeed, it has been suggested that an alteration in DDX3's subcellular localization could be associated with malignant transformation (Chao et al., 2006; Heerma van Voss et al., 2017b). However, one of these studies suggested that DDX3 is nuclear in untransformed (epidermal) cells and cytoplasmic in tumour cells (squamous cell carcinoma) (Chao et al., 2006), while a more recent study suggested that increased nuclear DDX3 (which was defined as being present in more than $1 \%$ of cells) correlates with worse patient outcome, larger tumour size, positive lymph nodes, higher grade cancers and (although not statistically significant) mitotic activity, in cohorts of colorectal and breast cancer patients (Heerma van Voss et al., 2017b). When we started this study, we had hoped to identify conditions that lead to increased nuclear accumulation of DDX3. During SeV infection, DDX3 becomes phosphorylated on multiple serine residues by the kinases
A

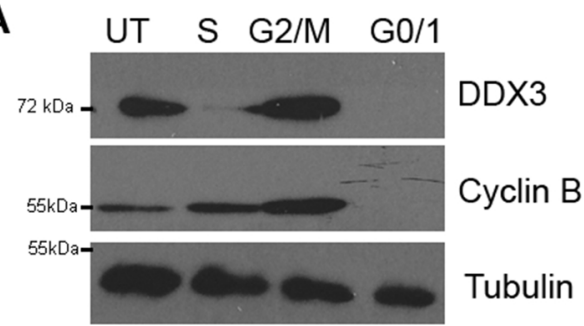

B

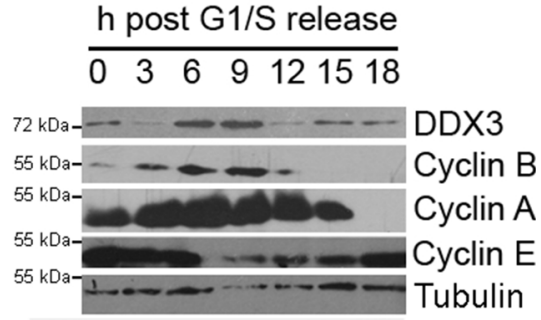

Fig. 7. DDX3 expression is upregulated during G2/M phase. A: HeLa cells were either left untreated or arrested at different cell cycle stages with thymidine (S-Phase), nocodazole (G2/M) or serum starvation (G0/1). Cell lysates were prepared from equal number of cells and subjected to SDS-PAGE and Western Blot Analysis with the indicated antibodies. B: Cells were first arrested in S-Phase with a double thymidine block and then released into normal growth medium. Samples for SDS-PAGE and

Western Blot analysis were collected every $3 \mathrm{~h}$ post release. Each subfigure is representative of two experiments. 
IKKe/TBK1 and it has been suggested to subsequently act as a transcriptional regulator of the IFN $\beta$ promoter (Gu et al., 2013; Soulat et al., 2008). The High Content analysis detected only a minor, but statistically significant increase in nuclear DDX3 $1 \mathrm{~h}$ and $24 \mathrm{~h}$ after infection. (Fig. 4A and B). Therefore, DDX3's role in the IFN induction pathway is either mainly cytoplasmic or nuclear retention of a small fraction of the overall cellular pool of DDX3 is sufficient to mediate its transcriptional regulation of the ifnb promoter. Unfortunately, due to the redundancy of the NLSs in DDX3, we were unable to generate an import-deficient point mutant of DDX3 that would have helped to clarify this question. Similarly, DDX3 has also been suggested to act in the DNA damage response (Sun et al., 2013), but exposure of cells to etoposide only induced minor changes in the nuclear-cytoplasmic distribution of DDX3 according to our HC analysis (Fig. 4). Thus, DDX3's nucleo-cytoplasmic distribution seemed to be largely refractory to various different cellular stimuli. In theory, what could cause nuclear accumulation of DDX3? Certainly, defects in the CRM-1 pathway or loss of DDX3's N-terminal NES would result in clear nuclear retention of DDX3 based on our data and also shown in Heerma van Voss et al. (2017b). Nuclear retention of DDX3 could also be caused by enhanced binding of DDX3 to nuclear proteins or chromatin, limiting its export via CRM-1 indirectly. While we failed to show regulation of DDX3's localization by phosphorylation at T204 and T323, other post-translational modifications of DDX3 could enhance its binding to nuclear factors, and thereby increase its nuclear localization. Could increased import rather than decreased export drive nuclear accumulation of DDX3? Theoretically this is certainly possible, especially given the fact that the NLSs in DDX3 appear to be rather weak. However, a recent study attempted to enhance nuclear import of DDX3 by attaching additional SV40-derived consensus NLSs. Surprisingly, this did not have a major effect on localization of DDX3, suggesting that export is extremely efficient and that reduced export is the key factor regulating nuclear accumulation of DDX3 in cases where this does occur (Heerma van Voss et al., 2017b).

\subsection{Alteration of DDX3's subcellular localization and expression level during mitosis}

We observed an increased accumulation of DDX3 in the nucleus during early mitosis, namely during prophase or prometaphase. This was visible in unsynchronized Hela cells where we phenotypically identified early mitotic cells, and further confirmed by staining of DDX3 in cells that were arrested in prometaphase by thymidine/nocodazole treatment (Fig. 5). In addition, western blot analysis demonstrated increased DDX3 protein levels during early mitosis. Presumably, this increase in (nuclear) DDX3 is linked to its role in centrosome regulation and chromosome segregation during mitosis, as described in two previous studies (Chen et al., 2017; Pek and Kai, 2011). Pek at al. suggested that DDX3 localises close to condensing chromosomes during prophase/prometaphase in HeLa cells (Pek and Kai, 2011), and the recent study by Chen et al. demonstrated co-localisation of DDX3 with centrosomes (Chen et al., 2017). Our images also suggest an association of DDX3 with the mitotic spindle during metaphase through to cytokinesis (Fig. 5B). Chen et al. also showed that knocking down DDX3 expression slows down progression of the cell cycle through G2/M phase (Chen et al., 2017), suggesting that increased expression and nuclear accumulation of DDX3 might be required for progressing cells through G2/M.

The mechanism behind the increased nuclear accumulation of DDX3 during mitosis is unclear. We had hypothesized that phosphorylation of DDX3 by Cyclin B, which is upregulated during early mitosis and has been shown to phosphorylate DDX3 at Thr204 and Thr323 (Sekiguchi et al., 2007), could mediate increased nuclear import or retention of DDX3. However, mutation of these two threonine residues to phosphomimetic aspartic acid did not induce nuclear accumulation of DDX3 (Fig. 6), thus we were unable to find evidence for this hypothesis. It would be interesting to address in future studies whether other post- translation modifications of DDX3 that occur in a cell-cycle dependent manner facilitate its nuclear retention.

Our observation that DDX3's expression and nuclear localization is increased during early mitosis should be relevant when interpreting DDX3 staining in tumour samples. Is it possible that increased nuclear DDX3 expression in tumour samples is due to the higher mitotic activity of tumour cells compared to normal tissue cells? In this context, it will be important to clarify in future studies whether increased nuclear DDX3 is a 'symptom' of cells entering mitosis, or whether nuclear DDX3 is an active driver of mitotic activity. It would be useful to identify effectors of DDX3's nuclear accumulation and to elucidate its downstream consequences.

\section{Conclusion}

In this paper we clarified that the conserved N-terminal NES is required for nuclear export of human DDX3 and identified at least three regions in DDX3 that contain independent NLSs. We also demonstrated that nuclear localisation and protein expression levels of DDX3 are increased during mitotic prophase, which could be linked to its proposed role in chromosome segregation and has relevance for a potential use of DDX3 expression as a biomarker in human cancers.

\section{Author contributions}

Conceived, designed and analysed the experiments: RB, LG, AHH, AL, VG and MS. Performed the experiments: RB with help from AHH and LG. Wrote the paper: RB and MS. All authors read and revised the final version of the manuscript.

\section{Competing financial interests}

The authors have no competing financial interests.

\section{Acknowledgements}

We gratefully acknowledge use of the facilities in The Irish National Center for High Content Screening and Analysis. Ruth Brennan was funded through the HRB Scholars Programme in Immunology ( $\mathrm{PhD} /$ 20007/9). HRB Ireland had no involvement in study design or data analysis and interpretation.

\section{Appendix A. Supplementary data}

Supplementary material related to this article can be found, in the online version, at doi:https://doi.org/10.1016/j.ejcb.2018.08.001.

\section{References}

Askjaer, P., Bachi, A., Wilm, M., Bischoff, F.R., Weeks, D.L., Ogniewski, V., Ohno, M., Niehrs, C., Kjems, J., Mattaj, I.W., Fornerod, M., 1999. RanGTP-regulated interactions of CRM1 with nucleoporins and a shuttling DEAD-Box helicase. Mol. Cell. Biol. $19,6276-6285$.

Askjaer, P., Rosendahl, R., Kjems, J., 2000. Nuclear export of the DEAD box An3 protein by CRM1 is coupled to An3 helicase activity. J. Biol. Chem. 275, 11561-11568.

Bol, G.M., Vesuna, F., Xie, M., Zeng, J., Aziz, K., Gandhi, N., Levine, A., Irving, A., Korz D., Tantravedi, S., Heerma van Voss, M.R., Gabrielson, K., Bordt, E.A., Polster, B.M., Cope, L., van der Groep, P., Kondaskar, A., Rudek, M.A., Hosmane, R.S., van der Wall, E., van Diest, P.J., Tran, P.T., Raman, V., 2015. Targeting DDX3 with a small molecule inhibitor for lung cancer therapy. EMBO Mol. Med. 7, 648-669.

Botlagunta, M., Vesuna, F., Mironchik, Y., Raman, A., Lisok, A., Winnard Jr., P., Mukadam, S., Van Diest, P., Chen, J.H., Farabaugh, P., Patel, A.H., Raman, V., 2008. Oncogenic role of DDX3 in breast cancer biogenesis. Oncogene 11, 11.

Boulon, S., Westman, B.J., Hutten, S., Boisvert, F.-M., Lamond, A.I., 2010. The nucleolus under stress. Mol. Cell 40, 216-227.

Chao, C.H., Chen, C.M., Cheng, P.L., Shih, J.W., Tsou, A.P., Lee, Y.H., 2006. DDX3, a DEAD box RNA helicase with tumor growth-suppressive property and transcriptional regulation activity of the $\mathrm{p} 21$ waf1/cip1 promoter, is a candidate tumor suppressor. Cancer Res. 66, 6579-6588.

Chen, W.J., Wang, W.T., Tsai, T.Y., Li, H.K., Lee, Y.W., 2017. DDX3 localizes to the centrosome and prevents multipolar mitosis by epigenetically and translationally 
modulating p53 expression. Sci. Rep. 7, 9411.

Cruciat, C.-M., Dolde, C., de Groot, R.E.A., Ohkawara, B., Reinhard, C., Korswagen, H.C., Niehrs, C., 2013. RNA helicase DDX3 is a regulatory subunit of casein kinase 1 in Wnt- $\beta$-catenin signaling. Science 339, 1436-1441.

Frohlich, A., Rojas-Araya, B., Pereira-Montecinos, C., Dellarossa, A., Toro-Ascuy, D., Prades-Perez, Y., Garcia-de-Gracia, F., Garces-Alday, A., Rubilar, P.S., ValienteEcheverria, F., Ohlmann, T., Soto-Rifo, R., 2016. DEAD-box RNA helicase DDX3 connects CRM1-dependent nuclear export and translation of the HIV-1 unspliced mRNA through its N-terminal domain. Biochim. Biophys. Acta 1859, 719-730.

Gu, L., Fullam, A., Brennan, R., Schröder, M., 2013. The human DEAD-box helicase 3 couples IKK-epsilon to IRF3 activation. Mol. Cell. Biol. 33, 2004-2015.

Gu, L., Fullam, A., McCormack, N., Höhn, Y., Schröder, M., 2017. DDX3 directly regulates TRAF3 ubiquitination and acts as a scaffold to co-ordinate assembly of signalling complexes downstream from MAVS. Biochem. J. 474, 571-587.

Guttler, T., Madl, T., Neumann, P., Deichsel, D., Corsini, L., Monecke, T., Ficner, R., Sattler, M., Gorlich, D., 2010. NES consensus redefined by structures of PKI-type and Rev-type nuclear export signals bound to CRM1. Nat. Struct. Mol. Biol. 17, $1367-1376$

Heerma van Voss, M.R., Brilliant, J.D., Vesuna, F., Bol, G.M., van der Wall, E., van Diest, P.J., Raman, V., 2017a. Combination treatment using DDX3 and PARP inhibitors induces synthetic lethality in BRCA1-proficient breast cancer. Med. Oncol. 34, 33.

Heerma van Voss, M.R., Vesuna, F., Bol, G.M., Afzal, J., Tantravedi, S., Bergman, Y., Kammers, K., Lehar, M., Malek, R., Ballew, M., Ter Hoeve, N., Abou, D., Thorek, D., Berlinicke, C., Yazdankhah, M., Sinha, D., Le, A., Abrahams, R., Tran, P.T., van Diest, P.J., Raman, V., 2018. Targeting mitochondrial translation by inhibiting DDX3: a novel radiosensitization strategy for cancer treatment. Oncogene 37 (1), 63-74.

Heerma van Voss, M.R., Vesuna, F., Bol, G.M., Meeldijk, J., Raman, A., Offerhaus, G.J., Buerger, H., Patel, A.H., van der Wall, E., van Diest, P.J., Raman, V., 2017b. Nuclear DDX3 expression predicts poor outcome in colorectal and breast cancer. Onco Targets Ther. 10, 3501-3513.

Hogbom, M., Collins, R., van den Berg, S., Jenvert, R.M., Karlberg, T., Kotenyova, T., Flores, A., Karlsson Hedestam, G.B., Schiavone, L.H., 2007. Crystal structure of conserved domains 1 and 2 of the human DEAD-box helicase DDX3X in complex with the mononucleotide AMP. J. Mol. Biol. 372, 150-159.

Kudo, N., Wolff, B., Sekimoto, T., Schreiner, E.P., Yoneda, Y., Yanagida, M., Horinouchi, S., Yoshida, M., 1998. Leptomycin B inhibition of signal-mediated nuclear export by direct binding to CRM1. Exp. Cell Res. 242, 540-547.

la Cour, T., Kiemer, L., Molgaard, A., Gupta, R., Skriver, K., Brunak, S., 2004. Analysis and prediction of leucine-rich nuclear export signals. Protein Eng. Des. Sel. 17, 527-536.

Lai, M.C., Lee, Y.H., Tarn, W.Y., 2008. The DEAD-box RNA helicase DDX3 associates with export mRNPs as well as TAP and participates in translational control. Mol. Biol. Cell $2,2$.

Lai, M.-C., Chang, W.-C., Shieh, S.-Y., Tarn, W.-Y., 2010. DDX3 Regulates cell growth through translational control of cyclin E1. Mol. Cell. Biol. 30, 5444-5453.

Lai, M.C., Sun, H.S., Wang, S.W., Tarn, W.Y., 2015. DDX3 functions in antiviral innate immunity through translational control of PACT. FEBS J. 283 (1), 88-101 (similar issue, epub in 2015).

Maga, G., Falchi, F., Radi, M., Botta, L., Casaluce, G., Bernardini, M., Irannejad, H., Manetti, F., Garbelli, A., Samuele, A., Zanoli, S., Este, J.A., Gonzalez, E., Zucca, E., Paolucci, S., Baldanti, F., De Rijck, J., Debyser, Z., Botta, M., 2011. Toward the discovery of novel anti-HIV drugs. Second-generation inhibitors of the cellular
ATPase DDX3 with improved anti-HIV activity: synthesis, structure-activity relationship analysis, cytotoxicity studies, and target validation. ChemMedChem 6, 1371-1389.

Mehdi, A.M., Sehgal, M.S., Kobe, B., Bailey, T.L., Boden, M., 2011. A probabilistic model of nuclear import of proteins. Bioinformatics 27, 1239-1246.

Nakai, K., Horton, P., 1999. PSORT: a program for detecting sorting signals in proteins and predicting their subcellular localization. Trends Biochem. Sci. 24, 34-36.

Oshiumi, H., Sakai, K., Matsumoto, M., Seya, T., 2010. DEAD/H BOX 3 (DDX3) helicase binds the RIG-I adaptor IPS-1 to up-regulate IFN-beta-inducing potential. Eur. J. Immunol. 40, 940-948.

Pek, J.W., Kai, T., 2011. DEAD-box RNA helicase Belle/DDX3 and the RNA interference pathway promote mitotic chromosome segregation. Proc. Natl. Acad. Sci. 108, 12007-12012.

Radi, M., Falchi, F., Garbelli, A., Samuele, A., Bernardo, V., Paolucci, S., Baldanti, F., Schenone, S., Manetti, F., Maga, G., Botta, M., 2012. Discovery of the first small molecule inhibitor of human DDX3 specifically designed to target the RNA binding site: towards the next generation HIV-1 inhibitors. Bioorg. Med. Chem. Lett. 22 2094-2098.

Schröder, M., 2010. Human DEAD-box protein 3 has multiple functions in gene regulation and cell cycle control and is a prime target for viral manipulation. Biochem. Pharmacol. 79, 297-306.

Schroder, M., Baran, M., Bowie, A.G., 2008. Viral targeting of DEAD box protein 3 reveals its role in TBK1/IKK-epsilon-mediated IRF activation. EMBO J. 17, 17.

Sekiguchi, T., Iida, H., Fukumura, J., Nishimoto, T., 2004. Human DDX3Y, the Y-encoded isoform of RNA helicase DDX3, rescues a hamster temperature-sensitive ET24 mutant cell line with a DDX3X mutation. Exp. Cell Res. 300, 213-222.

Sekiguchi, T., Kurihara, Y., Fukumura, J., 2007. Phosphorylation of threonine 204 of DEAD-box RNA helicase DDX3 by cyclin B/cdc2 in vitro. Biochem. Biophys. Res. Commun. 356, 668-673.

Sharma, D., Jankowsky, E., 2014. The Ded1/DDX3 subfamily of DEAD-box RNA helicases. Crit. Rev. Biochem. Mol. Biol. 49, 343-360.

Soto-Rifo, R., Rubilar, P.S., Limousin, T., de Breyne, S., Decimo, D., Ohlmann, T., 2012. DEAD-box protein DDX3 associates with eIF4F to promote translation of selected mRNAs. EMBO J. 31, 3745-3756.

Soto-Rifo, R., Rubilar, P.S., Ohlmann, T., 2013. The DEAD-box helicase DDX3 substitutes for the cap-binding protein eIF4E to promote compartmentalized translation initiation of the HIV-1 genomic RNA. Nucleic Acids Res. 41, 6286-6299.

Soulat, D., Burckstummer, T., Westermayer, S., Goncalves, A., Bauch, A., Stefanovic, A., Hantschel, O., Bennett, K.L., Decker, T., Superti-Furga, G., 2008. The DEAD-box helicase DDX3X is a critical component of the TANK-binding kinase 1-dependent innate immune response. EMBO J. 26, 26.

Spector, D.L., Lamond, A.I., 2011. Nuclear speckles. Cold Spring Harb. Perspect. Biol. 3.

Sun, M., Song, L., Li, Y., Zhou, T., Jope, R.S., 2008. Identification of an antiapoptotic protein complex at death receptors. Cell Death Differ. 15, 1887-1900.

Sun, M., Zhou, T., Jonasch, E., Jope, R.S., 2013. DDX3 regulates DNA damage-induced apoptosis and p53 stabilization. Biochim. Biophys. Acta 1833, 1489-1497.

Wagstaff, K.M., Jans, D.A., 2009. Importins and beyond: non-conventional nuclear transport mechanisms. Traffic 10, 1188-1198.

Yedavalli, V.S., Neuveut, C., Chi, Y.H., Kleiman, L., Jeang, K.T., 2004. Requirement of DDX3 DEAD box RNA helicase for HIV-1 Rev-RRE export function. Cell 119, $381-392$. 